\section{Umweltberichte: notwendig, aber nicht hinreichend}

\author{
Ein Instrument zum Aufbau von Dialog und Kooperation \\ mit Anspruchsgruppen
}

Kraft Jacobs Suchard Deutschland hat 1994/95 seinen ersten Umweltbericht veröffentlicht (1). Eines der Grundprinzipien des Umweltmanagements bei Kraft Jacobs Suchard und damit erklärtes Ziel des Umweltberichtes ist das "Fordern und Fördern " des umweltpolitischen Dialogs und der umweltpolitischen Kooperation mit allen gesellschaftlichen Gruppen $(S .9,13,19)$. Die bisherigen Erfahrungen sind positiv bis vielversprechend - mit einer relativ deutlichen Ausnahme: die Umweltund Verbraucherverbände und auch die Wissenschaft. Diese halten sich mit einer Reaktion (noch) spürbar zurück (2).

\section{Vorgeschichte und Ziele}

Anfang 1992 wurde - damals noch bei Jacobs Suchard - ein umfassendes, integriertes Umweltmanagement-Konzept entwickelt, das seitdem Schritt für Schritt umgesetzt wird. Dieses Konzept sah die Erstellung eines Umweltberichtes bereits vor. Er sollte das Unternehmen nach innen und außen ökologisch transparent machen und so eine der wesentlichen Voraussetzungen für Dialog und Kooperation legen. Nachdem die für einen solchen Bericht notwendigen Vorarbeiten (3) sowie die Integration zum neuen Unternehmen Kraft Jacobs Suchard erfolgreich abgeschlossen waren, konnte er Ende 1994 fertiggestellt werden. Der Bericht sollte vor allem

- als möglichst umfassende Dokumentation von Positionen, Zielen, Projekten, Stärken und Schwächen der unternehmensinternen »ökologischen Standortbestimmung « dienen;

- alle Mitarbeiterinnen und Mitarbeiter informieren und so die interne Kommunikation und Kooperation verbessern und

- als Basisinstrument für die Kommunikation mit Externen dienen.

Um diese Anforderungen zu erfüllen, mußte der Bericht »state of the art« sein, also möglichst die von IÖW und future e. V. entwickelten Kriterien erfüllen (4). Obwohl das nicht immer möglich war und auch nicht immer gelungen ist, hat das IÖW in einem von Kraft Jacobs Suchard in Auftrag gegebenen Gutachten den Bericht grundsätzlich positiv bewertet (5) und der gerade erschienene »Unternehmenstester « (6) bezeichnet ihn sogar als vorbildlich.

\section{Zielgruppen}

Die wichtigste Zielgruppe waren und sind die Angehörigen des Unternehmens. Sie haben den Bericht deshalb auch zuerst, nämlich Ende 1994, bekommen. Ende Januar 1995 erfolgte dann sukzessive die Aussendung an die weiteren Zielgruppen, nämlich:

- die Wirtschaft, insbesondere den Handel, Lieferanten und andere Geschäftspartner des Unternehmens, Wirtschaftsverbände und
Kammern sowie andere Industrieunternehmen,

- Politik und Verwaltung, vor allem die Umweltministerien, Landesanstalten oder -ämter, das UBA und die Standortgemeinden des Unternehmens,

\section{- Umwelt- und Verbraucherverbände,}

- sonstige Meinungsbildner oder Multiplikatoren wie Universitäten und Institute.

Keine direkte Zielgruppe waren Medien und Verbraucher: Es wurde keinerlei aktive Medienarbeit betrieben und auch die Verbraucher wurden nicht direkt angesprochen (etwa über die Schaltung von Anzeigen). Beiden wurde der Bericht jeweils nur auf Anforderung geschickt. Neben Kostengründen waren dafür vor allem

Erstens ist nach Einschätzung von Kraft Jacobs Suchard im Bereich Umweltschutz die indirekte Kommunikation über Anspruchsgruppen zwar erheblich langsamer, aber wesentlich »nachhaltiger « als eine Medienkampagne und zweitens kann nach vorliegenden Erfahrungen von einem großen Interesse der Verbraucher an Umweltberichten von Unternehmen z. Zt. nicht ausgegangen werden.

Letzteres gilt erst recht für einen 48 -seitigen Bericht mit relativ großem Datenteil, der sehr wahrscheinlich nur von relativ wenigen Interessierten wirklich durchgelesen wird. Aus diesem Grund hat das Unternehmen eine kurze $\mathrm{Zu}$ sammenfassung in Form eines Leporellos erKommunikation zusätzlich ein etwa 9-minütiges Video als »Film zum Buch » produziert. Hier zeichnet sich der allgemeine Widerspruch zwischen Anspruch und Forderung nach möglichst umfassender Information einerseits und der »Informationsüberflutung « mit anschließender »Informationsaufnahmeverweigerung « ab, der durch »zielgruppenspezifische « Berichterstattung nur sehr begrenzt gelöst werden kann (wieviele Berichte müßte ein Unternehmen dann veröffentlichen?). - Vielleicht bieten hier die neuen, "interaktiven « elektronischen Medien einen Ausweg, weil Sie eine Kombination verschiedener Medien und das individuelle, »bedarfsgerechte« Abrufen von Informationen ermöglichen. zwei Überlegungen ausschlaggebend: stellt und für Zwecke der internen und externen

\section{Erste Erfahrungen}

Die bisherigen Erfahrungen sind sicher noch vorläufig, aber die Tendenz ist eindeutig positiv und in vielen Bereichen besser als erwartet. Intern sind vor allem zwei sich gegenseitig stützende und befruchtende Entwicklungen, festzustellen: Zum einen steht das Management seit Erscheinen des Berichtes noch eindeutiger zu den dort dokumentierten Inhalten und Zielen. Umweltpolitische Themen werden häufiger, engagierter und offensiver diskutiert - sicher auch eine Folge der Tatsache, daß die lange und teilweise sehr hitzige umweltpolitische Grundsatzdiskussion durch das Erscheinen des ersten Berichtes (endlich) abgeschlossen ist und nun auf einer anderen, nämlich allgemein akzeptierten Grundlage diskutiert werden kann.

Ein ähnlicher Prozeß findet zum anderen bei den Mitarbeiterinnen und Mitarbeitern statt. Zwar wurde der Bericht zunächst von einem Großteil nicht oder kaum zur Kenntnis genommen. Aber ein ebenfalls erheblicher Teil der Belegschaft - vor allem diejenigen, die an den verschiedenen Umweltprojekten beteiligt waren und sind - hat ihn sehr positiv aufgenommen, nimmt zunehmend auf ihn Bezug und macht ihn so auch für andere zu einem Gesprächsthema. Auch unter den Mitarbeitern wachsen konstruktive Kritik, Engagement und Kreativität in Sachen Umweltschutz.

\section{Positive Reaktionen aus Wirtschaft und Politik}

Auch die externen Reaktionen sind überwiegend positiv: Uneingeschränkt gilt das für die Wirtschaft, von der wir nur Zustimmung erhalten haben. Außerdem hat der Bericht sowohl zur Intensivierung bestehender als auch zur Entstehung neuer Kontakte und Kooperationen -z. T. über Branchengrenzen hinweg - geführt. Insbesondere die Zusammenarbeit mit den Handelspartnern von Kraft Jacobs Suchard hat sich noch weiter verbessert und bereits zur konkreten Planung neuer gemeinsamer Umweltprojekte geführt. Eine ähnlich positve Entwicklung zeichnet sich - wenn auch noch nicht so konkret und ausgeprägt - im Verhältnis zur Politik ab: Die bisherigen Signale aus wenigstens zwei Bundesländern stimmen optimistisch, wobei in diesem Fall die seit Juli 1993 bestehende, sehr erfolgreiche Kooperation zwischen Kraft Jacobs Suchard und dem Bremer Senat (S. 14 f.) wohl eine wichtigere Rolle spielt als der Bericht.

Aus der Wissenschaft gibt es dagegen bisher kaum Reaktionen. Zwar ist die Nachfrage ähnlich wie bei Agenturen und Consultants - erstaunlich hoch. Dennoch gibt es bis auf zwei Ausnahmen noch keine Beurteilung oder Meinungsäußerung aus diesem Bereich. Offensichtlich haben die an erstaunlich vielen Universitäten und Instituten laufenden Projekte zur Umweltberichterstattung noch nicht zu konkreten (Zwischen-) Ergebnissen geführt. 
Auf die Reaktion der »anspruchsvollsten Anspruchsgruppen « und Standardsetzer war das Unternehmen gespannt. Die weitgehende "Funkstille « im Bereich der Umwelt- und Verbraucherorganisationen war deshalb auf den ersten Blick besonders enttäuschend. Allerdings nur auf den ersten Blick. Denn für die - bisherige - Zurückhaltung gibt es eine ganze Reihe plausibler Gründe:

- Das auf beiden »Seiten « lange vorherrschende Lagerdenken ist nur schrittweise zu überwinden.

- Es gibt - ebenfalls auf beiden Seiten - die durchaus berechtigte Angst, von der jeweils anderen Seite vereinnahmt und über den Tisch gezogen zu werden - mit der Folge eines Profil- oder gar Identitätsverlustes. Die klare Abgrenzung beider Seiten hat ja auch den sehr positiven Effekt, kreatives Potential zu erzeugen, was durch einen zu engen Dialog oder gar Kooperation leicht verwässert werden kann.

- Sowohl Unternehmen als auch Umwelt- und Verbraucherverbände stehen im Wettbewerb. Eine klare Positionierung zum Wettbewerber ist deshalb (überlebens-)wichtig; ein zu »grünes « Image kann für Unternehmen, ein zu »wirtschaftfreundliches« für Anspruchsgruppen von erheblichem Nachteil sein.

- Die »Themenkonjunkturen« beider Seiten verlaufen nicht parallel, sondern versetzt: Die Existenzberechtigung und der "gesellschaftliche Wert« von Umwelt- und Verbraucherverbänden u. a. Anspruchsgruppen besteht ja zu einem großen Teil darin, schneller zu sein als Gesellschaft, Politik, Wirtschaft und oft auch Wissenschaft. Sie schaffen oder besetzen zukünftige Themen, die dann, wenn sie sich als gesellschaftliches Thema durchgesetzt haben, von den anderen gesellschaftlichen Akteuren erst en detail »abgearbeitet" werden müssen - und in dieser Zeit schaffen die Anspruchsgruppen neue Themen. Der Zeitraum, in dem alle Seiten parallel diskutieren, ist damit relativ kurz.

- Schließlich gibt es die sehr banalen, aber um so schlagenderen knappen Ressourcen. Dialog und Kooperation kosten beide Seiten Zeit und Geld und das ist auf beiden Seiten knapp, vor allem aber bei den Anspruchsgruppen. Effizientes Ressourcenmanagement wird deshalb versuchen, die Wege so kurz wie möglich und den Dialog so konkret wie möglich zu machen: Der Dialog über ein konkretes Problem oder Projekt zwischen Partnern an einem Ort ist um Größenordnungen effizienter als ein allgemeiner Dialog zwischen zwei Partnern, die $500 \mathrm{~km}$ voneinander entfernt sitzen.

\section{Resümee}

Die geschilderte positive Reaktion von Externen wirkt sich natürlich positiv auf die interne Ent- wicklung aus und umgekehrt. Zusammen haben diese Entwicklungen in der Tat das Potential, zu einem wenigstens teilweise »selbstorganisierenden Prozeß « zu werden, wobei ein - guter Umweltbericht sicher nicht alleinige Ursache ist, aber doch eine wichtige Katalysatorfunktion erfüllen kann.

Für eine dauerhaft positive Entwicklung letztlich entscheidend ist jedoch immer der nächste

1) Kraft Jacobs Suchard Deutschland, Umweltbericht 1991 -1995, erhältlich über: Kraft Jacobs Suchard Deutschland, Environment \& Associations, Langemarckstraße 4-20, 28199 Bremen; Tel. : 0421 / 599 - 3867, Fax: -3899). Im folgenden beziehen sich Seitenzahlen in Klammern auf diesen Bericht.

2) Eine klare Ausnahme von der Ausnahme ist der BUND, insbesondere der Landesverband Bremen, mit dem seit über drei Jahren ein sehr fruchtbarer Dialog besteht.

3) Vor allem die Definition von Grundpositionen und Zielen sowie der Aufbau eines ÖkoControlling-Systems und die Erstellung einer Input-Output-Analyse.

4) Vgl. Clausen, J.; Fichter, Klaus: Wissen-
Umweltbericht: Er bestimmt die interne und externe Diskussion, die Ziele und die konkreten Maßnahmen, bevor er veröffentlicht wird. Der zweite Umweltbericht von Kraft Jacobs Suchard Deutschland erscheint 1996 - das Unternehmen befindet sich ab sofort in der entscheidenden Phase.

Heiko Richert, Bremen

schaftlicher Endbericht Umweltweltberichterstattung

5) Erreicht wurden knapp 284 von 500 Punkten (entspr. 57\%) und wäre (zum Zeitpunkt der Erstellung des Gutachtens: Januar 1994) im IÖWRanking der fünftbeste deutsche Umweltbericht gewesen. Das Gutachten und die Bewertung sind nach Meinung des Unternehmens fair und zutreffend. Die festgestellten Mängel sollen möglichst im nächsten Bericht (1996) behoben werden.

6) imug - Institut für Markt - Umwelt - Gesellschaft u. a. (Hg. ), Der Unternehmenstester. Die Lebensmittelbranche, Reinbek bei Hamburg 1995 , S. 187 f.

\section{Umwelterklärungen als Instrument dialogorientierten Managements - Anforderungen der Öffentlichkeit}

Das Verhältnis der Unternehmen zur Öffentlichkeit ist in der Betriebswirtschaftslehre traditionell ein Teilgebiet der Marketinglehre. Public Relations umfassen die Kommunikationsbeziehungen zwischen einem Unternehmen und der nach Teilgruppen gegliederten Öffentlichkeit. Gleichwohl hat die Ausrichtung auf die Anforderungen der nichtmarktlichen Anspruchsgruppen in den Betrieben noch keineswegs den Stellenwert der Kundenorientierung erreicht. Noch immer ist die PR-Abteilung im wesentlichen als Stabsstelle der Unternehmensleitung zugegliedert; ohne Durchgriff und zumeist auch ohne Einfluß auf unternehmenspolitische Entscheidungen und letztlich auf die reaktive Erhaltung von Handlungsspielräumen kapriziert (1). Die ernsthafte Kommunikation mit umweltbezogenen Anspruchsgruppen ist heute im besten Fall Stückwerk, das aus dem Interesse einzelner aufgeschlossener Manager entsteht. Umgekehrt bleibt auch das Interesse der gesellschaftlichen Anspruchsgruppen am Umweltverhalten der Unternehmen einzelfallorientiert und ohne systematische Einbindung.

Angesichts der negativen Erfahrungen aus der Geschichte der Sozialbilanzierung (2) und dem offenkundigen Widerwillen gegen öffentliche Transparenz auch bei ansonsten umweltpolitisch aktiven Unternehmen ist es ein spannendes gesellschaftliches Experiment, ob die Umwelterklärung im Rahmen der EU-Ökoaudit-Verordnung die Basis für eine systematische, dialogorientierte betriebliche Umweltpolitik bilden kann.

\section{Informationsbedürfnisse der gesellschaftlichen Anspruchsgruppen}

In der EU-Verordnung zum Umweltmanagement und zur Umweltbetriebsprüfung kommt der Transparenz der Unternehmen gegenüber der Öffentlichkeit eine herausgehobene Bedeutung zu. Die Umwelterklärung stellt dazu das zentrale Element der Verordnung dar. Auf indirektem Weg sollen neue Informationstatbestände und neue Informationsflüsse erzeugt werden, die ihrerseits Marktkräfte im Sinne des Umweltschutzes induzieren (3).

Soll die Umwelterklärung ihre Aufgabe erfüllen, so ist zu berücksichtigen, welche Informationsbedürfnisse die gesellschaftlichen Anspruchsgruppen als Adressaten der Umwelterklärung haben. Voraussetzung ist, daß es sich um berechtigte, sinnvolle Ansprüche handelt. Probleme ergeben sich dabei aus dem Umstand, daß der Adressatenkreis heterogen sein kann, sehr unterschiedliche Interessen verfolgt und entsprechend unterschiedliche Informationsan- 
(c) 20I0 Authors; licensee IÖW and oekom verlag. This is an article distributed under the terms of the Creative Commons Attribution Non-Commercial No Derivates License (http://creativecommons.org/licenses/by-nc-nd/3.o/), which permits unrestricted use, distribution, and reproduction in any medium, provided the original work is properly cited. 